\title{
Fatores de risco associados ao prognóstico de adultos internados com pneumonia adquirida na comunidade ${ }^{1}$
}

\author{
Risk factors that influence the prognosis of \\ community-acquired pneumonia \\ in hospitalized adults
}

Maria Rita DONALISIO²

Bertha Siqueira Bernardi de OLIVEIRA²

Carlos Henrique Mamud ARCA²

June Barreiros FREIRE ${ }^{3}$

Daniela de Oliveira MAGRO²

RE S U M O

\section{Objetivo}

Avaliar parâmetros nutricionais no prognóstico de adultos internados em hospital geral, com pneumonia adquirida na comunidade.

\section{Métodos}

Foram estudados prospectivamente 61 casos da doença e analisadas variáveis: sociodemográficas, morbidade, hábitos de vida, antropométricas (índice de massa corporal, circunferência da cintura, porcentagem de gordura, porcentagem de perda de peso), bioquímicas (ureia, creatinina, albumina no momento da internação e no $18^{\circ}$ dia e pré-albumina (na internação, quarto, oitavo e $18^{\circ}$ dia) e evolução (menos de 10, mais de 10 dias de internação e/ou óbito).

\section{Resultados}

As variáveis antropométricas apresentaram-se diferentes entre os sexos, porém não se associaram com a má evolução da doença. Os níveis séricos de albumina e de pré-albumina encontraram-se baixos na internação em $80,3 \%$ e $86,9 \%$ dos casos, respectivamente. Após ajuste logístico, o hábito de fumar $(\mathrm{OR}: 1,23, \mathrm{IC}: 1,0-12,1)$,

\footnotetext{
1 Artigo elaborado a partir da dissertação de BSB OLIVEIRA, intitulada "Aspectos nutricionais em adultos internados com pneumonia adquirida na comunidade em hospital geral". Universidade Estadual de Campinas; 2010. Apoio: Fundação de Amparo à Pesquisa do Estado de São Paulo - Auxílio à Pesquisa, processo/FAPESP: 05/01553-7.

${ }^{2}$ Universidade Estadual de Campinas, Faculdade de Ciências Médicas, Departamento de Medicina Preventiva e Social. R. Tessália Vieira de Camargo, 126, 13083-887, Campinas, SP, Brasil. Correspondência para/Correspondence to: MR DONALISIO. E-mail: <rita.donalisio@gmail.com>.

${ }^{3}$ Hospital Estadual Sumaré. Sumaré, SP, Brasil.
} 
a presença de mais de uma imagem ou derrame pleural no exame radiológico de tórax (OR: 1,4 IC: 1,24-15,3) e a pré-albumina baixa no quarto dia $(\mathrm{OR}: 6,1 \mathrm{IC}: 1,7-22,5)$ foram preditores de má evolução do quadro de pacientes com pneumonia adquirida na comunidade.

\section{Conclusão}

Indicador bioquímico nutricional como a pré-albumina, o tabagismo e mais de uma imagem no exame radiológico de tórax são parâmetros de gravidade de pneumonia úteis na condução clínica de infecções.

Termos de indexação: Nutrição. Pneumonia. Pré-albumina. Risco.

\section{A B S T R A C T}

\section{Objective}

The present study assessed the influence of nutritional parameters on the prognosis of community-acquired pneumonia in adults admitted to a general hospital.

\section{Methods}

A total of 61 individuals with community-acquired pneumonia were studied prospectively and the following data analyzed: sociodemographic variables, morbidity, life habits, anthropometric variables (body mass index, waist circumference, percentage of body fat, percentage of weight lost), biochemical variables (urea, creatinine, albumin on admission and 18 days later, prealbumin on admission and 4, 8 and 18 days later), and outcome (hospital stay shorter than 10 days, longer than 10 days and/or death).

\section{Results}

Anthropometric variables differed between genders but were not associated with poor outcome. Albumin and prealbumin levels were low on admission in $80.3 \%$ and $86.9 \%$ of the cases, respectively. Logistic regression showed that smoking (OR:1.23; Cl:1.0-12.1), more than one finding or pleural effusion on chest radiographs (OR:1.4; Cl:1.24-15.3) and low prealbumin on day 4 (OR:6.1; Cl:1.7-22.5) predicted a poor outcome for patients with community-acquired pneumonia.

\section{Conclusion}

Nutrition-related biochemical markers, such as low prealbumin, smoking and more than one finding on chest radiographs, indicate severe pneumonia and are useful for deciding treatment strategy.

Indexings terms: Nutrition. Pneumonia. Prealbumin. Risk.

\section{N T R O D U ÇÃ O}

A pneumonia é uma doença grave associada à significativa morbidade e mortalidade em adultos. Pneumonia Adquirida na Comunidade (PAC) é aquela que acomete o paciente fora do ambiente hospitalar ou que surge nas primeiras 48 horas da admissão ${ }^{1}$. Cerca de $80 \%$ dos casos de PAC podem ser tratados em ambulatório, porém os pacientes internados apresentam taxas de mortalidade entre $12 \%$ e $25 \%^{1,2}$.

A pneumonia é registrada como a segunda causa de hospitalizações no Brasil, respondendo por $11,8 \%$ das internações na região Sudeste, em $2007^{3}$. Os casos são mais frequentes nos meses de inverno, entre os menores de cinco e maiores de 60 anos $^{1,2}$.
Alguns estudos têm mostrado a variedade de preditores de risco de morte e hospitalização prolongada entre os casos de PAC ${ }^{2,4,5}$. A maior gravidade da doença é observada em idosos, indivíduos institucionalizados, com antecedente de alcoolismo e portadores de doenças crônicas (doença pulmonar crônica, cardiopatias, diabetes Mellitus, insuficiência renal, insuficiência hepática, entre outras) ${ }^{1,6-8}$.

Um aspecto relevante na evolução e na gravidade dos casos de pneumonia, particularmente em idosos, é o estado nutricional. O fornecimento adequado de energia, de proteínas, de vitaminas e de minerais tem papel crítico na resposta imune e, portanto, interfere na suscetibilidade a infecções, particularmente em países 
em desenvolvimento, onde a má-nutrição é comum ${ }^{6,7}$.

Tanto os indicadores clássicos (Índice de Massa Corporal - IMC) e pregas subcutâneas $)^{9}$ como os do estado imunológico (imunoglobulinas A, M e G) e os prognósticos (albumina, transferrina, pré-albumina, proteína C-reativa) têm sido associados com a evolução clínica de doenças crônicas, de infecções e após intervenções cirúrgicas $^{8,10-15}$

Embora seja crescente o número de estudos sobre aspectos nutricionais clínicos, antropométricos e bioquímicos, são poucos os que tratam desses parâmetros em pacientes com infecções pulmonares ${ }^{11,14}$, particularmente em países em desenvolvimento. A avaliação da evolução clínica da PAC, considerando-se aspectos nutricionais, pode trazer informações relevantes sobre o comportamento desses parâmetros na população brasileira, submetida às condições de vida e saúde específicas.

Este estudo tem como objetivo investigar alguns parâmetros nutricionais, clínicos e radiológicos no prognóstico de adultos com PAC internados no Hospital Estadual Sumaré (HES) no período de 24 meses.

\section{M É T O D OS}

Trata-se de estudo prospectivo sobre aspectos nutricionais em pacientes internados no Hospital Estadual Sumaré com diagnóstico de PAC ( $n=61$ ) no período de outubro de 2005 a setembro de 2007. O hospital tem 226 leitos - 34 na unidade de clínica médica -, e é referência para a microrregião de Sumaré (SP), que tem 630 mil habitantes. É certificado como nível III (máximo) pela Organização Nacional de Acreditação (ONA), que expressa avaliação hospitalar dentro de parâmetros pré-estabelecidos, do uso de dispositivos ou ferramentas da gestão da qualidade.

Como critério de inclusão foram considerados os indivíduos maiores de 14 anos, com diagnóstico de pneumonia (presença de um ou mais sintomas e imagem sugestiva em exame radiológico simples de tórax), que não tomam medicamentos imunossupressores, e consentiram em participar da pesquisa. Foram excluídos da amostra pacientes com infecção pelo HIV/Aids, sequelados, com demência e os encaminhados com história de internação em outro serviço nos últimos 15 dias. Este estudo foi aprovado pelo Comitê de Ética em Pesquisa da Faculdade de Ciências Médicas da Unicamp, protocolo $n^{\circ} 241 / 2005$ ).

Os pacientes foram submetidos a exame clínico e avaliação nutricional por nutricionista treinado e foram coletadas informações sobre antecedentes e hábitos de vida. Os exames radiológicos simples de tórax foram analisados por um único radiologista.

As variáveis analisadas foram sociodemográficas (sexo, idade, anos de escolaridade), comorbidades, hábitos de vida, medidas antropométricas (peso, altura, índice de massa corporal, circunferência da cintura, percentuais de gordura corporal e de massa livre de gordura), exames bioquímicos (creatinina, ureia, albumina, pré-albumina), tipo de imagem na radiografia simples de tórax (única, mais de uma, derrame pleural), além de evolução clínica (internação até 10 dias e 10 dias ou mais e/ou óbito).

Foi considerado "tabagista" o indivíduo que referiu fumar todos os dias, independentemente da quantidade. Considerou-se exposição ao álcool o consumo de mais de uma garrafa de bebida não destilada por dia ou duas ou mais doses de destilados. Em relação aos parâmetros nutricionais, considerou-se desnutrido o paciente com IMC < 18,5kg/m²; eutrófico o paciente com IMC entre 18,5 e $24,9 \mathrm{~kg} / \mathrm{m}^{2}$; com sobrepeso o paciente com IMC entre 25 e $29,9 \mathrm{~kg} / \mathrm{m}^{2}$; e com obesidade o IMC $\left.\geq 30 \mathrm{~kg} / \mathrm{m}^{2}\right)^{9}$. Os parâmetros para a circun-ferência de cintura foram analisados segundo as faixas de risco; para o sexo masculino: ideal abaixo de $94 \mathrm{~cm}$, risco moderado de 94 a $102 \mathrm{~cm}$ e risco grave $>102 \mathrm{~cm}$; para as mulheres: ideal abaixo de $80 \mathrm{~cm}$, risco moderado de $80 \mathrm{a}$ $88 \mathrm{~cm}$ e risco grave $>88 \mathrm{~cm}^{16}$. 
A composição corporal por bioimpedância elétrica teve como parâmetros as seguintes faixas de percentuais de gordura: para homens - risco $(\leq 5 \%)$, abaixo da média e média ( $6 \%$ a $15 \%)$, acima da média ( $16 \%$ a $24 \%$ ) e risco $(\geq 25 \%)$; para mulheres - risco ( $\leq 8 \%)$, abaixo da média e média (9\% a 23\%), acima da média (24\% a 31\%) e risco $(\geq 32 \%)^{19}$. Os pontos de corte das medidas de reatância e biorresistência obtidos pela bioimpedância tetrapolar foram $<60$ ohms e $>400$ ohms, respectivamente, tendo sido considerados adequados para avaliar indiretamente a retenção de água extracelular associada ao processo inflamatório ${ }^{18}$. Foi questionado ao pacientes seu peso habitual nos últimos seis meses e no último mês, para se analisar uma possível perda de peso de $5 \%$ no último mês e de $10 \%$ nos últimos seis meses.

As dosagens de albumina e pré-albumina foram realizadas no momento da internação para identificar o perfil proteico. A dosagem de albumina foi repetida após 17 a 20 dias e as de pré-albumina repetidas após quatro, oito e dezoito dias de internação ${ }^{19}$. Os valores de referência de normalidade adotados para a albumina foram de 3,5 a 5,0 $\mathrm{g} / \mathrm{dL}$ e para a pré-albumina de 0,2 a $0,4 \mathrm{~g} / \mathrm{L}$ e realizados pelo laboratório do Hospital Estadual Sumaré.

Quanto à análise estatística, as proporções de casos segundo variáveis nutricionais foram comparadas utilizando-se o teste Qui-quadrado. Foram analisadas as medidas de associação Odds Ratio (OR) referentes às diversas exposições entre casos com evolução desfavorável (pacientes que evoluíram para óbito e/ou internação maior que 10 dias) e casos com evolução favorável (pacientes sobreviventes com alta antes de 10 dias). Após teste bivariado de associação pelo OR e Qui-quadrado, foi ajustado modelo de regressão logística múltipla não condicional, tendo-se como variável a resposta à evolução clínica do caso (grave/não grave).

Foram selecionadas para o modelo todas as variáveis que mostraram associação com a variável resposta (evolução desfavorável) em nível de significância de $20 \%(p<0,20)$, permanecendo no modelo múltiplo aquelas que apresentaram $p<0,05$. A força da associação entre as variáveis independentes e a dependente foi expressa em valores estimados de Odds Ratio (brutos e ajustados) com intervalo de confiança de 95\%.

O programa Epi Info versão $6.04 \mathrm{~b}$ e Microsoft Office Excel 2007 foram empregados para a construção do banco de dados. Os cálculos foram feitos por meio do programa estatístico $R$ versão 2.11.1.

\section{RES ULTADOS}

Houve predomínio de PAC em homens $33(54,1 \%)$, em maiores de 40 anos - 44 ( $72,1 \%)$, tendo $22(36,1 \%)$ indivíduos menos de quatro anos de escolaridade, e a maioria instrução até o primeiro grau completo $(78,7 \%)$. A exposição ao álcool foi significativamente diferente entre os sexos; com relação ao tabagismo houve diferença, porém não estatisticamente significante $(p=0,08)$, entre os $16(51,6 \%)$ homens e $7(25,9 \%)$ mulheres fumantes no momento da internação. Não houve diferença significante entre a prevalência de comorbidades entre os sexos, embora as mulheres apresentassem percentuais maiores em todas as doenças crônicas investigadas, com exceção das doenças renais. As principais doenças foram hipertensão arterial, diabetes e cardiopatia, com 50,0\%, 25,0\% e 25,0\%, respectivamente. Por outro lado, os homens mostraram maiores prevalências de exposições prévias a poeiras - 7 (25\%), ao fumo - 16 (51,6\%) e ao álcool - 12 $(37,5 \%)$, este último significativamente maior nesse grupo (Tabela 1).

O tempo de internação médio foi de 13,6 dias (min. 3 e máx. 60 dias), sendo a letalidade de 4,9\% entre os casos analisados. A circunferência da cintura e o percentual de gordura foram diferentes entre os sexos $(p<0,05)$. As mulheres apresentaram maiores percentuais de medidas que indicam risco, como a circunferência de cintura acima do ideal - 20 (71,4\%). Das mulheres, $17(60,7 \%)$ estavam com o peso acima do ideal, enquanto $11(33,4 \%)$ homens encontravam-se com sobrepeso ou obesidade (Tabela 2). 
Tabela 1. Perfil sociodemográfico, hábitos de vida e antecedentes de morbidade dos pacientes com pneumonia internados no Hospital Estadual de Sumaré entre outubro de 2005 e setembro de 2007. Sumaré (SP).

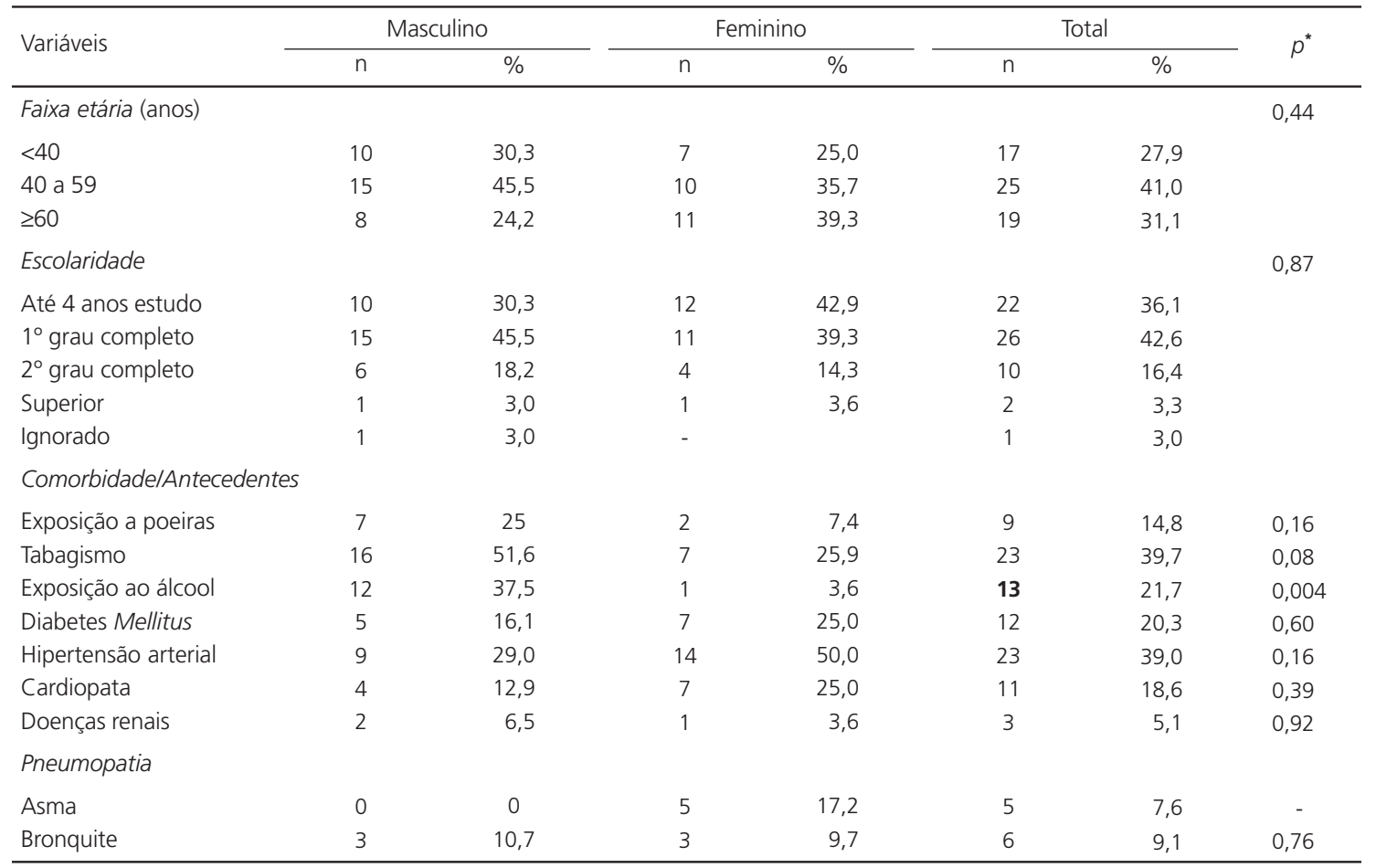

*Valor $p$ em teste Qui-quadrado com correção de Yates. Em negrito a variável com valor significativo $(p \leq 0,05)$.

Tabela 2. Variáveis nutricionais dos pacientes com pneumonia internados no Hospital Estadual de Sumaré entre outubro de 2005 e setembro de 2007. Sumaré (SP).

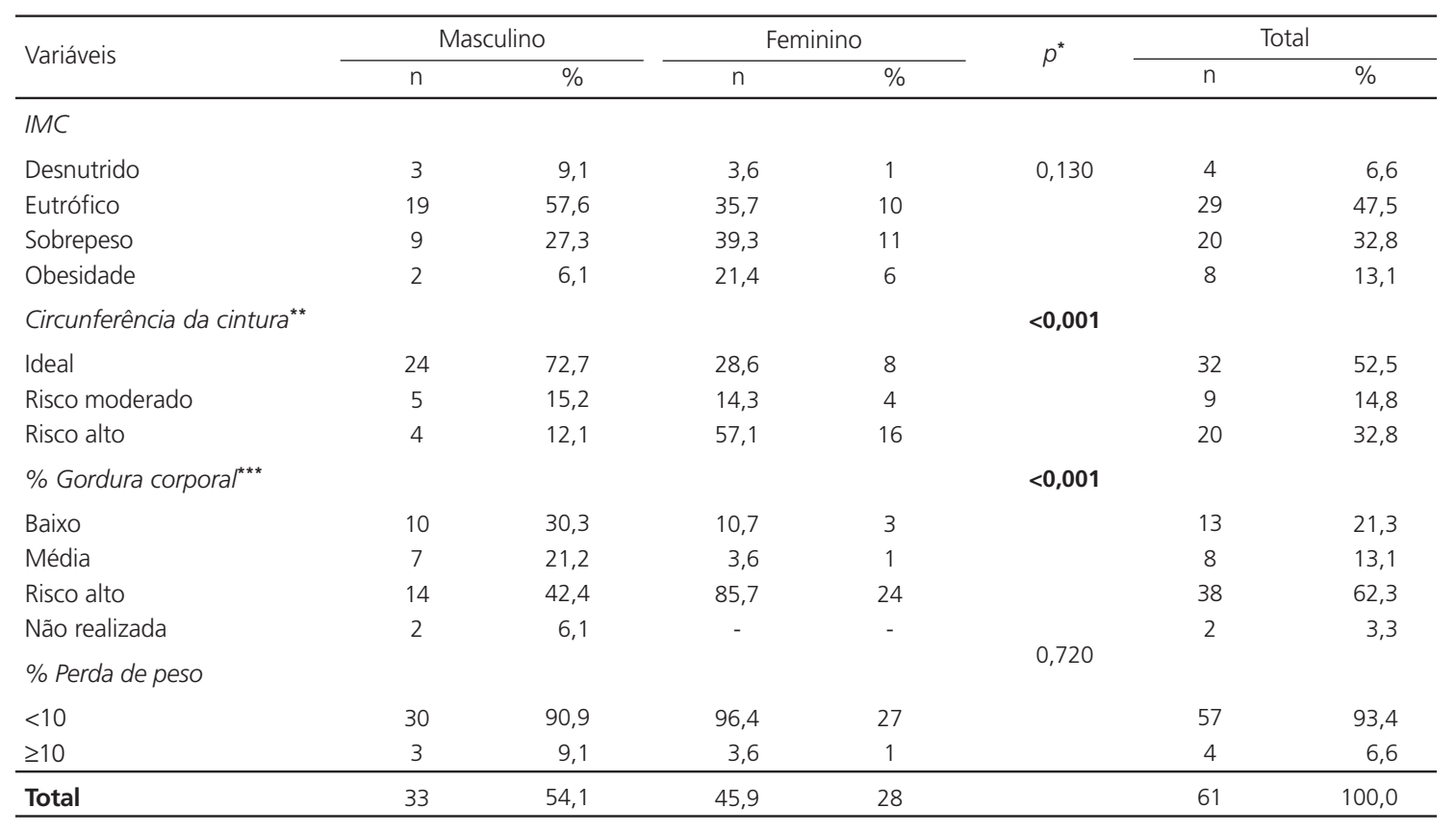

*Valor $p$ em teste Qui-quadrado com correção de Yates; ${ }^{* *}$ Circunferência cintura: ideal: homem: $<94 \mathrm{~cm}$ e mulher $\leq 80 \mathrm{~cm}$; risco moderado: homem: 94 - 102cm; mulher: 80 - 88cm; risco alto: homem: $\geq 102 \mathrm{~cm}$ e mulher: $\geq 88 \mathrm{~cm}$; ${ }^{* * *} \%$ Gordura corporal (Bioimpedância): baixa - homem: $\leq 5$ e mulher $\leq 8$; média - homem $>5$ e $\leq 15$ e mulher $>8$ e $\leq 23$; risco - homem: $>15$ e mulher: $\geq 24$. IMC: Índice de Massa Corporal; PAC: Pneumonia Adquirida na Comunidade; Em negrito as variáveis com valores significativos $(p \leq 0,05)$. 
Em relação ao IMC e à evolução clínica de PAC, não houve diferença significante entre os casos de maior ou menor gravidade (Tabela 3). Dos 61 pacientes estudados, 21 (34,4\%) apresentaram obesidade; destes, apenas 5 (18,5\%) evoluíram com mais de 10 dias de internação ou morreram.

Embora os exames de pacientes com alta hospitalar não tenham sido realizados, os níveis de pré-albumina e albumina mantiveram-se baixos na maioria dos pacientes no decorrer da internação, como é de se esperar em quadros infecciosos (Tabela 4). Entre os pacientes com evolução desfavorável, 23 (85,2\%) apresentaram hipopré-albuminemia no momento da internação; destes, 20 (88\%) mantiveram níveis baixos de pré-albumina após o quarto dia de internação. Embora a deficiência apresentada seja maior nos casos graves, cerca de dois terços dos casos não graves também apresentaram níveis séricos abaixo do esperado. No decorrer do seguimento, as medidas de pré-albumina mantiveram-se baixas nos casos graves, com tendência à melhora nos casos não graves.

As medidas antropométricas e nutricionais obtidas na admissão do paciente não se mostraram parâmetros de risco para a gravidade do caso, isto é, não foram preditores de má evolução clínica da PAC na análise univariada $(p>0,20)$. Por outro lado, a dosagem de pré-albumina no quarto dia de internação, mais de uma imagem na radiografia simples de tórax e o hábito de fumar mantiveram-se significativos no modelo logístico múltiplo, constituindo-se preditores de risco nesses pacientes (Tabela 5).

Tabela 3. Classificação do estado nutricional dos pacientes com pneumonia internados no Hospital Estadual de Sumaré entre outubro de 2005 e setembro de 2007, segundo o IMC e evolução clínica. Sumaré (SP).

\begin{tabular}{|c|c|c|c|c|c|c|}
\hline \multirow{2}{*}{ 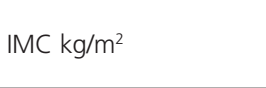 } & \multicolumn{2}{|c|}{ Caso graves* } & \multicolumn{2}{|c|}{ Não graves** } & \multicolumn{2}{|c|}{ Total } \\
\hline & $n$ & $\%$ & $n$ & $\%$ & $n$ & $\%$ \\
\hline Baixo $(<18,5)$ & 2 & 7,4 & 2 & 5,9 & 4 & 6,6 \\
\hline Eutrófico $(18,5$ a 24,9$)$ & 14 & 51,9 & 15 & 44,1 & 29 & 47,5 \\
\hline Sobrepeso $(25$ a 29,9$)$ & 6 & 22,2 & 14 & 41,2 & 20 & 32,8 \\
\hline Obesidade $(\geq 30)$ & 5 & 18,5 & 3 & 8,8 & 21 & 34,4 \\
\hline Total & 27 & 44,3 & 34 & 55,7 & 61 & 100,0 \\
\hline
\end{tabular}

* Graves: internação >10 dias e/ou óbito; * * Não graves: internação $\leq 10$ dias.

Teste Qui-quadrado=3,26; GL=3; $p=0,39 ;$ IMC: Índice de Massa Corporal; PAC: Pneumonia Adquirida na Comunidade.

Tabela 4. Distribuição dos níveis séricos de albumina e pré-albumina nos dias 0, 4, 8 e 18 de internação, dos pacientes com pneumonia internados no Hospital Estadual de Sumaré entre outubro de 2005 e setembro de 2007. Sumaré (SP).

\begin{tabular}{|c|c|c|c|c|c|c|c|c|}
\hline \multirow{2}{*}{ Níveis séricos } & \multicolumn{2}{|c|}{ Dia $0^{*}$} & \multicolumn{2}{|c|}{ Dia 4} & \multicolumn{2}{|c|}{ Dia 8} & \multicolumn{2}{|c|}{ Dia 18} \\
\hline & $\mathrm{n}$ & $\%$ & $\mathrm{n}$ & $\%$ & $\mathrm{n}$ & $\%$ & $n$ & $\%$ \\
\hline \multicolumn{9}{|c|}{ Albumina (g/dL) } \\
\hline$<3,5$ & 49 & 80,3 & - & - & - & - & 16 & 61,5 \\
\hline $3,5-5,0$ & 12 & 19,7 & - & - & - & - & 10 & 38,5 \\
\hline Total & 61 & 100,0 & - & - & - & - & 26 & 100,0 \\
\hline \multicolumn{9}{|c|}{ Pré-albumina ( $g / L)$} \\
\hline$<0,2$ & 53 & 86,9 & 29 & 65,9 & 10 & 72,0 & 2 & 66,7 \\
\hline $0,2-0,4$ & 8 & 13,1 & 15 & 34,1 & 3 & 23,1 & 1 & 33,3 \\
\hline Total & 61 & 100,0 & 44 & 100,0 & 13 & 100,0 & 3 & 100,0 \\
\hline
\end{tabular}

Dia 0 = dia de internação do paciente; PAC: Pneumonia Adquirida na Comunidade. 
Tabela 5. Estatísticas obtidas de ajuste de modelo de regressão logística univariada e múltipla entre casos de pneumonia adquirida na comunidade (caso = evolução desfavorável ${ }^{*}$ ) e variáveis demográficas, morbidade e nutricionais, internados no Hospital Estadual de Sumaré, entre outubro de 2005 e setembro de 2007. Sumaré (SP).

\begin{tabular}{|c|c|c|c|c|c|}
\hline Variáveis & $\mathrm{OR}_{\text {bruto }}$ & IC 95\% & $p^{* *}$ & $\mathrm{OR}_{\text {ajustado }}$ & IC 95\% \\
\hline Sexo (masculino) & 1,5 & $0,5-4,1$ & 0,43 & & \\
\hline Idade & 0,9 & $0,9-1,0$ & 0,56 & & \\
\hline Tabagismo & 3,4 & $0,9-12,1$ & 0,05 & 1,2 & $1,1-12,2$ \\
\hline Exposição ao álcool & 2,0 & $0,5-7,7$ & 0,38 & & \\
\hline Doença crônica (pelo menos 1) & 1,4 & $0,5-4,1$ & 0,51 & & \\
\hline $\mathrm{IMC}>30 \mathrm{~kg} / \mathrm{m}^{2}$ & 3,2 & $0,9-13,2$ & 0,09 & & \\
\hline Perda peso > $5 \%$ & 0,7 & $0,2-2,8$ & 0,74 & & \\
\hline Circunferência cintura masculino (>94cm) & 1,3 & $0,2-7,7$ & 0,91 & & \\
\hline Circunferência cintura feminino (>80cm) & 1,9 & $0,3-13,6$ & 0,75 & & \\
\hline Resistência <400ohms & 1,3 & $0,5-3,2$ & 0,80 & & \\
\hline Reactância <60ohms & 0,8 & $0,2-2,5$ & 0,78 & & \\
\hline$\%$ de gordura corporal masculino (>15\%) & 0,7 & $0,1-4,4$ & 0,96 & & \\
\hline$\%$ de gordura corporal feminino (>24\%) & 0,5 & $0,1-2,9$ & 0,56 & & \\
\hline Atividade física* & 0,8 & $0,3-2,1$ & 0,82 & & \\
\hline $1^{\text {a }}$ Albumina (baixa) & 12,4 & $2,2-236,1$ & 0,02 & & \\
\hline $1^{\circ}$ dia Pré-albumina (baixa) & 0,8 & $0,1-4,3$ & 0,79 & & \\
\hline $4^{\circ}$ ao $7^{\circ}$ dia Pré-albumina (baixa) & 3,7 & $0,9-18,9$ & 0,07 & 6,1 & $1,7-22,5$ \\
\hline 15 a $18^{\circ}$ dia Albumina (baixa) & 2,3 & $0,5-10,7$ & 0,33 & & \\
\hline $18^{\circ}$ dia Pré-albumina (baixa) & 1,2 & $0,1-34,1$ & 0,54 & & \\
\hline Raios-X tórax ${ }^{* * *}$ & 2,9 & $1,1-9,0$ & 0,04 & 1,4 & $1,2-15,3$ \\
\hline Creatinina & 3,8 & $1,1-15,5$ & 0,05 & & \\
\hline
\end{tabular}

*Evolução desfavorável: internação por mais de 10 dias e/ou óbito; ${ }^{* *}$ Valor do p obtido pelo teste Qui-quadrado; ${ }^{* * *}$ Raios-X de tórax com mais de uma imagem e/ou derrame pleural. Em negrito as variáveis com valores significativos $(p \leq 0,05)$.

\section{I S C U S S Ã O}

Este estudo analisou a totalidade dos pacientes hospitalizados com PAC e avaliou as características clínicas e nutricionais durante o período de 24 meses. Identificou a hábito de fumar, a presença de mais de uma imagem na radiografia simples de tórax e a dosagem da pré-albumina no quarto dia de internação como preditores de evolução desfavorável dos casos, no modelo final de regressão logística múltipla.

A detecção de mais de uma imagem ou de derrame pleural no primeiro exame de Raios- $X$ de tórax na ocasião de internação do paciente foi preditor de risco de má evolução clínica da PAC neste estudo, como também observam outros autores'.

As variáveis socioeconômicas não se associaram de forma significativa com o maior tem- po de internação tampouco com a mortalidade. Vale ressaltar que a maioria dos indivíduos com PAC tinha baixa escolaridade, além de comorbidades e alta exposição ao tabagismo e ao álcool, entre os homens. Isso pode ser explicado pelo fato de que quanto mais baixa a renda, maior a proporção da população dependente do Sistema Único de Saúde ${ }^{17,20}$.

A alta prevalência de fumantes entre os internados quando comparada à população ge$\mathrm{ral}^{21}$ pode estar associada ao maior risco de doenças pulmonares nesses indivíduos. Estudos longitudinais mostram aumento da mortalidade por pneumonia nos fumantes associado ao número de cigarros fumados e ao tempo total desse hábito de vida. Tabagistas de mais de 20 cigarros por dia têm risco três vezes maior de adquirir pneumonia do que os que nunca fumaram, não havendo diferença de risco usando cigarros de filtro ou com diferentes profundidades de inalação ${ }^{4}$. 
No presente estudo, observaram-se altas prevalências de sobrepeso e obesidade nas mulheres com PAC $(60,7 \%)$ e nos homens $(33,4 \%)$ sem relação com a gravidade do caso. Nos pacientes internados, essa prevalência foi superior à observada em estudo da população urbana da região Sul e Sudeste $(50,4 \%)$ e nas capitais brasileiras onde o excesso de peso e a obesidade aumentam com a idade até 54 anos entre homens e até 64 anos entre as mulheres ${ }^{22}$. Embora o sobrepeso e a obesidade estejam associados a várias doenças crônicas, como hipertensão arterial, doenças cardiovasculares, osteoartrite, diabetes tipo II e alguns tipos de câncer ${ }^{21,23}$, neste estudo essa relação não foi encontrada, possivelmente devido à pequena casuística.

A avaliação antropométrica apresentou limitações principalmente por não existir testes padronizados para estudos comparativos entre os indicadores. Embora haja um consenso de que uma perda de peso maior que 10,0\% nos últimos seis meses seja um parâmetro de avaliação de morbidade e mortalidade, essa abordagem apresenta limitações, uma vez que outras variáveis podem interferir nesses resultados, como as associadas ao gênero e às condições sociais dos pacientes. Encontraram-se 5 (8,0\%) pacientes com perda de peso maior que $10,0 \%$ e $18,2 \%$ com perda de 5,0\% de peso no último mês. Essa variável não foi associada ao agravamento da PAC.

A circunferência da cintura e o percentual de gordura corporal apresentaram-se diferentes entre os sexos, porém sem relação com a gravidade de PAC. Parâmetros nutricionais têm sido incorporados às atividades clínicas de rotina durante a internação hospitalar, particularmente as associadas com a função pulmonar ${ }^{4}$. O sobrepeso, a obesidade e a circunferência da cintura podem prejudicar a função e a estrutura respiratória devido à limitação de expansão torácica pela concentração de gordura abdominal, causando apneia, que pode se associar à hipóxia cíclica, complicando-se com a obesidade e a hipertensão $5,8,24$.
Riquelme et al. ${ }^{14}$ mostraram clara relação entre aspectos nutricionais e mau prognóstico dos pacientes com pneumonia comunitária, o que indica que há maior risco de morte naqueles com menor percentual de massa muscular. Isso não foi observado no presente estudo também possivelmente devido ao pequeno número de casos.

Da mesma forma que outras variáveis nutricionais, as alterações nos níveis de albumina não se relacionaram de forma significante com o agravamento de PAC, embora a maioria dos pacientes tenha apresentado deficiência no momento da internação.

A hipoalbuminemia se associa com a morbidade e a mortalidade: pacientes com massa celular corpórea e níveis de albumina sérica reduzidos apresentam maior probabilidade de morrer por pneumonia do que pacientes sem comprometimento nutricional ${ }^{8}$. No entanto, essa proteína apresenta meia vida elevada, podendo não ser um indicador sensível para identificar desnutrição aguda e sim desequilibro nutricional crônico. Além disso, a albumina tem baixa especificidade, pois seus níveis estão alterados em várias doenças, embora tenha grande utilidade em estudos populacionais de doenças crônicas ${ }^{12,14}$.

Por outro lado, a pré-albumina mostra uma associação estatisticamente significativa com complicações infecciosas e mortes por infecções no modelo de regressão logística final, como sugerido em outros estudos em circunstâncias clínicas diversas ${ }^{8,11,12,14,15,25}$. Neste estudo, observou-se alta prevalência de anormalidades dessa proteína já no momento da internação na maioria dos pacientes - 51 (84\%). Notou-se, porém, que os que continuaram apresentando deficiência de pré-albumina após quatro dias de internação foram os que permaneceram mais que 10 dias internados ou foram a óbito.

A pré-albumina tem uma meia vida curta (mais ou menos dois dias) e é a primeira proteína a mostrar níveis alterados na desnutrição aguda. Nesses casos, é um marcador precoce e sensível desse tipo de desnutrição, pois seus níveis voltam ao normal tão logo aconteça a reposição nutri- 
cional ${ }^{11,19}$. Bernstein et al. ${ }^{25}$ consideram a pré-albumina o melhor parâmetro para avaliação nutricional clínica.

As internações de pneumonias de origem comunitária são devidas, principalmente, ao maior risco de complicações clínicas e morte do paciente; vários manuais clínicos discutem escores de gravidade para auxiliar o médico no momento de internação ${ }^{1,2}$. Na região de estudo, ocorre muitas vezes a internação por motivos sociais, isto é, devido à dificuldade dos pacientes em seguir as orientações terapêuticas, em retornar para reavaliação clínica, além da frequente precariedade da rede ambulatorial para o seguimento desses pacientes, entre outras. Vale destacar que pacientes jovens conseguem internação mais rápida em outros hospitais da região, vindo para o HES um "perfil mais idoso" ou de mais difícil manejo clínico.

Muitas dessas internações de PAC no HES podem ter sido influenciadas por esses motivos, dificultando a identificação de variáveis, particularmente antropométricas e nutricionais, preditoras de riscos de complicações clínicas, como apontadas por outros autores 1,5,24,26. Estudos com maior casuística e classificação clínica das PAC, segundo escores clínicos de gravidade², poderiam esclarecer a influência dessas variáveis nutricionais nesses pacientes.

\section{O N CLUS Ã O}

O derrame pleural e/ou a presença de mais de uma imagem no exame de Raios- $X$ de tórax, a baixa dosagem de pré-albumina no quarto dia de internação e o tabagismo foram associados, neste estudo, à má evolução clínica das PAC. Destaca-se a pré-albumina como um indicador nutricional independente, capaz de predizer a ocorrência de complicações e a maior permanência do paciente no hospital.

\section{COLABORA D ORES}

MR DONALISIO planejou o trabalho, participou da coleta, análise de dados e discussão dos resultados e elaboração do manuscrito. BSB OLIVEIRA e CHM ARCA participaram da coleta de dados, montagem do banco de dados, análise e discussão dos resultados. JB FREIRE e DO MAGRO participaram da organização das atividades de campo, análise, discussão de resultados e elaboração do manuscrito.

\section{REFERÊ NCIAS}

1. Corrêa RA, Lundgren LFC, Pereira-Silva JL, Silva RLF, Cardoso AP, Lemos ACM, et al. Diretrizes brasileiras para pneumonia adquirida na comunidade em adultos imunocompetentes: 2009. J Bras Pneumol. 2009; 35(6):574-601.

2. Fine MJ, Auble TE, Yealy DM, Hanusa BH, Weissfeld LA, Singer DE. A prediction rule to identify low-risk patients with community-acquired pneumonia. N Engl J Med. 1997; 336(44):243-50.

3. Brasil. Sistema Único de Saúde. Departamento de Informática do SUS. Morbidade Hospitalar do SUS: por local de residência - Brasil. Brasília: Ministério da Saúde [acesso 2010 jul]. Disponível em: <http:// tabnet.datasus.gov.br/cgi/tabcgi.exe?sih/cnv/ mruf.def>.

4. Gomes L. Fatores de risco e medidas profiláticas nas pneumonias adquiridas na comunidade. J Pneumol. 2001; 27(2):97-113.

5. Mandell LA, Wunderink RG, Anzueto A, Bartlett JG, Campbell GD, Dean NC, et al. Infectious Diseases Society of America/American Thoracic Society consensus guidelines on the management of community-acquired pneumonia in adults. Clin Infect Dis. 2007; 44(Suppl 2):27-72.

6. Calder PC, Samantha K. The immune system: a target for functional foods? Br J Nutr. 2002; 88: S165-76.

7. Katona P, Katona J.The interaction between nutrition and infection. Clin Infect Dis. 2011; 46(10): 1582-8.

8. Hedlund J, Hansson LO, Ortqvist A. Short and long term prognosis for middle-aged and elderly patients hospitalized with pneumonia: impact of nutritional and inflammatory factors. Scand J Infect Dis. 1995; 27(1):32-7.

9. Grant JP, Custer PB, Thurlow J. Current techniques of nutritional assessment. Surg Clin North Am. $1981 ; 61(3): 437-63$

10. Sachs E, Bernstein LH. Protein markers of malnutrition status as related to sex and age. Clin Chem. 1986; 32:339-41.

11. Junqueira JCS, Soares EC, Correa Filho HR, Hoehr NF, Magro DO, Ueno M. Nutritional risk factors for 
postoperative complications in Brazilian elderly patients undergoing major elective surgery. Nutrition. 2003; 19:321-6.

12. Kurpad AV. The requirements of protein, and amino acid during acute and chronic infections. Indian J Med Res. 2006; 124:129-48.

13. Loeb MB. Community-acquired pneumonia in older people: the need for a broader perspective. JAGS. 2003; 51:539-43.

14. Riquelme RO, Riquelme MO, Rioseco MLZ, Cárdenas G, Torres C. Neumonía adquirida en la comunidad en el anciano hospitalizado: aspectos clínicos y nutricionales. Rev Méd Chile. 2008; 136:587-93.

15. Ingenbleek $Y$, van Den Schrieck HG, Nayer P, De Visscher M. Albumin, transferrin and the thyroxine-binding prealbumin/retinol-binding protein (TBPA-RBP) complex in assessment of malnutrition. Clin Chim Acta. 1975; 63:61-7.

16. Callaway CW, Chumlea WC, Bouchard C. Circunferences. In: Lohman TG, Roche AF, Martorell $\mathrm{R}$, editors. Anthropometric standardization reference manual. Champaign: Human Kinetics Books; 1988.

17. Oliveira EXG, Travassos C, Carvalho MS. Acesso à internação hospitalar nos municípios brasileiros em 2000: territórios do Sistema Único de Saúde. Cad Saúde Pública. 2004; 20(Supl 2):298-309.

18. Heyward VH, Stolarczyk LM. Método de impedância bioelétrica. In: Heyward VH, Stolarczyk LM. Avaliação da composição corporal aplicada. São Paulo: Manole; 2000

19. Bottoni A, Oliveira GPC, Ferrini MT, Waitzberg DL. Avaliação nutricional: exames laboratoriais. In: Waitzberg DI. Nutrição oral, enteral e parenteral na prática clínica. $3^{a}$ ed. São Paulo: Atheneu; 2000.
20. Brasil. Ministério da Saúde. Secretaria de Vigilância em Saúde. Vigitel Brasil 2009: vigilância de fatores de risco e proteção por doenças crônicas por inquérito telefônico. Brasília: MS; 2010.

21. Abrantes MM, Lamounier JA, Colosimo EA. Prevalência de sobrepeso e obesidade nas regiões Nordeste e Sudeste do Brasil. Rev Assoc Med Bras. 2003, 49(2):162-6.

22. Tavares EL, Anjos LA. Perfil antropométrico da população idosa brasileira. Resultados da Pesquisa Nacional sobre Saúde e Nutrição. Cad Saúde Pública. 1999; 15(4):759-68.

23. Gigante DP, Moura EC, Sardinha LMV. Prevalência de excesso de peso e obesidade e fatores associados, Brasil, 2006. Rev Saúde Pública. 2009; 43(Supl 2):83-89.

24. Borel JC, Roux-Lombard P, Tamisier R, Arnaud C, Monneret D, Arnol N, et al. Endothelial dysfunction and specific inflammation in obesity hypoventilation syndrome. PLoS One. 2009; 4(8):e6733. doi: 10.13 71/journal.pone.0006733.

25. Bernstein L, Bachman T, Meguid M, Ament M, Baumgartner T, Kinosian B, et al. Measurement of visceral protein status in assessing protein and energy malnutrition: standard of care. Nutrition. 1995; 11:169-71.

26. Donalisio MR, Arca CHM, Madureira P. Perfil clínico, epidemiológico e etiológico de pacientes com pneumonia adquirida na comunidade internados em um hospital geral da microrregião de Sumaré, SP. J Bras Pneumol. 2011; 37(2):200-8.

Recebido em: 20/4/2011

Versão final em: 16/12/2011

Aprovado em: 23/3/2012 